\title{
TWISTED BURNSIDE-FROBENIUS THEORY FOR ENDOMORPHISMS OF POLYCYCLIC GROUPS
}

\author{
ALEXANDER FEL'SHTYN AND EVGENIJ TROITSKY
}

\begin{abstract}
Let $R(\varphi)$ be the number of $\varphi$-conjugacy (or Reidemeister) classes of an endomorphism $\varphi$ of a group $G$. We prove for several classes of groups (including polycyclic) that the number $R(\varphi)$ is equal to the number of fixed points of the induced map of an appropriate subspace of the unitary dual space $\widehat{G}$, when $R(\varphi)<\infty$. Applying the result to iterations of $\varphi$ we obtain Gauss congruences for Reidemeister numbers.

In contrast with the case of automorphisms, studied previously, we have a plenty of examples having the above finiteness condition, even among groups with $R_{\infty}$ property.
\end{abstract}

\section{INTRODUCTION}

The Reidemeister number or $\varphi$-conjugacy number of an endomorphism $\varphi$ of a group $G$ is the number of its Reidemeister or $\varphi$-conjugacy classes, defined by the equivalence

$$
g \sim x g \varphi\left(x^{-1}\right) .
$$

The interest in twisted conjugacy relations has its origins, in particular, in the NielsenReidemeister fixed point theory (see, e.g. [29, 30, 6]), in Arthur-Selberg theory (see, e.g. $[42,1]$ ), Algebraic Geometry (see, e.g. [27]), and Galois cohomology (see, e.g. [41]). In representation theory twisted conjugacy probably occurs first in [23] (see, e.g. [44, 37]).

An important problem in the field is to identify the Reidemeister numbers with numbers of fixed points on an appropriate space in a way respecting iterations. This opens possibility of obtaining congruences for Reidemeister numbers and other important information.

For the role of the above "appropriate space" typically some versions of unitary dual can be taken. This desired construction is called the twisted Burnside-Frobenius theory (TBFT), because in the case of a finite group and identity automorphism we arrive to the classical Burnside-Frobenius theorem on enumerating of (usual) conjugacy classes.

In the present paper we prove TBFT for endomorphisms of any polycyclic group (Theorem 6.1) and Gauss congruences for Reidemeister numbers.

In the case of automorphism this problem was solved for polycyclic-by-finite groups in $[13,21]$. Preliminary and related results, examples and counter-examples can be found in $[8,5,6,12,14,9,11,45,46]$.

For endomorphisms of polycyclic fundamental groups of infra-solvmanifolds of type (R) the Gauss congruences were obtained in [19].

2000 Mathematics Subject Classification. 20C; 20E45; 22D10; 37C25; 47H10; 55M20.

Key words and phrases. Reidemeister number, twisted conjugacy class, Burnside-Frobenius theorem, unitary dual, matrix coefficient, rational (finite) representation, Gauss congruences, surface group.

The work of A.F. is partially supported by the grant 346300 for IMPAN from the Simons Foundation and the matching 2015-2019 Polish MNiSW fund.

The work of E.T. is partially supported by the Russian Foundation for Basic Research under grant 1601-00357. 
The importance of obtaining the present results namely for endomorphisms is justified by a plenty of examples (in contrast with the case of automorphisms) (see, in particular, 1.1, 1.4 , and 6.4 below).

A related fact is that, in contrast with TBFT for automorphisms, TBFT for endomorphisms is weakly connected with the theory of $R_{\infty}$-groups (see e.g. Example 6.4). A group is called $R_{\infty}$ if any its automorphism has infinite Reidemeister number. This was the subject of an intensive recent research and for many groups this property was established, see the following partial bibliography and the literature therein: $[7,33,15,16,10,2,25,17,36$, $34,18,26,40,4,20,28]$. In some situations the property $R_{\infty}$ has some direct topological consequences (see e.g. [26]).

Decision problems for twisted conjugacy classes of endomorphisms of polycyclic groups were studied in [39].

The paper is organized in the following way.

In Section 1 we show how drastically the theory of twisted conjugacy classes for endomorphisms differs from the theory for automorphisms.

In Section 2 we introduce and investigate a dual object for a pair $(G, \varphi)$.

In Section 3 we prove Gauss congruences for Reidemeister numbers.

In Section 4 we discuss proof of twisted Burnside-Frobenius theorem for endomorphisms of any finite group.

Section 5 is technical.

In Section 6 we proof the twisted Burnside-Frobenius theorem for endomorphisms of polycyclic groups. We finish the paper with a series of examples of $R_{\infty}$ groups admitting endomorphisms with finite Reidemeister numbers .

ACKNOWLEDGEMEnT: This work is a part of our joint research project at the Max-Planck Institute for Mathematics (Bonn) and the most part of the results were obtained there in Spring 2017.

The work of A.F. is partially supported by the grant 346300 for IMPAN from the Simons Foundation and the matching 2015-2019 Polish MNiSW fund.

The work of E.T. is partially supported by the Russian Foundation for Basic Research under grant 16-01-00357.

\section{Preliminaries}

First of all, let us make the following observation, showing how drastically the Reidemeister numbers world for endomorphisms differs from the Reidemeister world for automorphisms.

Proposition 1.1. For any group $G$ there exists an endomorphism $\varphi: G \rightarrow G$ with $R(\varphi)<$ $\infty$, namely $R(\varphi)=1$.

Proof. Take $\varphi$ to be the trivial map $\varphi(g)=e$ for any $g \in G$.

This observation can be enforced.

Proposition 1.2. Suppose, $\varphi: G \rightarrow G$ is an endomorphism and $K:=\operatorname{Ker} \varphi$. Then all Reidemeister classes are some unions of $K$-cosets.

Proof. Let $g_{1}$ and $g_{2}$ be in a $K$-coset, i.e. $g_{1} g_{2}^{-1}=k \in K$. Then $g_{1}=k g_{2}=k g_{2} \varphi\left(k^{-1}\right)$.

Using Lemma 1.9 we immediately obtain

Corollary 1.3. The map $p_{\varphi}: G \rightarrow G / K$ gives a bijection of Reidemeister classes. 
Corollary 1.4. Any endomorphism with finite image has a finite Reidemeister number.

Definition 1.5. Denote by $\widehat{G}$ the unitary dual of $G$, by $\widehat{G}_{f}$ the part of the unitary dual formed by irreducible finite-dimensional representations, and by $\widehat{G}_{f f}$ the part of $\widehat{G}_{f}$ formed by finite representations, i.e. representations that factorize through a finite group.

Definition 1.6. Let us call o.t. commutant the operator theoretical commutant of a set $D$ of bounded operators on a Hilbert space, i.e. the subset of the algebra of all bounded operators on this space, formed by all operators that commute with all elements of $D$. Denote it $D^{\star}$.

Lemma 1.7. A representation is irreducible if and only if the o.t. commutant of the set of representing operators is formed just by scalar operators [31, Theorem 2, p. 114].

In particular, if $\rho$ is irreducible and $\varphi$ is an epimorphism, $\rho \circ \varphi$ is also irreducible.

Lemma 1.8. If representations $\pi$ and $\rho$ of $G$ are equivalent, then $\pi \circ \varphi$ and $\rho \circ \varphi$ are equivalent for any endomorphism $\varphi: G \rightarrow G$.

Proof. Indeed: use the same intertwining operator.

The following statement is well known

Lemma 1.9. Suppose, $\varphi: G \rightarrow G$ is an endomorphism and $H \subset G$ is a normal $\varphi$-invariant subgroup, then $p: G \rightarrow G / H$ induces an epimorphism of Reidemeister classes.

Proof. Indeed, suppose, $p\left(g^{\prime}\right)=p(\widetilde{g}) p(g) p\left(\varphi\left(\widetilde{g}^{-1}\right)\right)$. Then it is equal to $p\left(\widetilde{g} g \varphi\left(\widetilde{g}^{-1}\right)\right)$.

Also, we need the following

Lemma 1.10. Any Reidemeister class of $\varphi$ is $\varphi$-invariant.

Proof. Indeed, $\varphi(x)=x^{-1} x \varphi(x)$.

The following fact can be extracted from [24, Prop. 1.6].

Lemma 1.11. In the above situation $R\left(\left.\varphi\right|_{H}\right) \leqslant R(\varphi) \cdot\left|C\left(\varphi_{G / H}\right)\right|$ where $C\left(\varphi_{G / H}\right)$ is the fixed point subgroup for the induced map $\varphi_{G / H}: G / H \rightarrow G / H$.

The following statement is well known in the field.

Lemma 1.12. A right shift by $g \in G$ maps bijectively Reidemeister classes of $\varphi$ onto Reidemeister classes of $\tau_{g^{-1}} \circ \varphi$, where $\tau_{g}$ is the inner automorphism: $\tau_{g}(x)=g x g^{-1}$.

Proof. This follows immediately from the equality

$$
x y \varphi\left(x^{-1}\right) g=x(y g) g^{-1} \varphi\left(x^{-1}\right) g=x(y g)\left(\tau_{g^{-1}} \circ \varphi\right)\left(x^{-1}\right) .
$$

\section{DuAl OBJECT FOR A PAIR $(G, \varphi)$}

For an automorphism or at least epimorphism $\varphi$ and an irreducible representation $\rho$, the representation $\rho \circ \varphi$ is always irreducible. For a general endomorphism and a finitedimensional $\rho$ one can only decompose the representation into irreducible components. So, one obtains a sort of multi-valued mapping and the corresponding dynamical system is not defined. To avoid this problem we will act as follows. 
Definition 2.1. We will call a representation class $[\rho]$ a $\widehat{\varphi}$-f-point, if $\rho$ is equivalent to $\rho \circ \varphi$ (we avoid to say that it is a fixed point, because we can not define the corresponding dynamical system).

Definition 2.2. An element $[\rho] \in \widehat{G}$ (respectively, in $\widehat{G}_{f}$ or $\widehat{G}_{f f}$ ) is called $\varphi$-irreducible if $\rho \circ \varphi^{n}$ is irreducible for any $n=0,1,2, \ldots$

Denote the corresponding subspaces of $\widehat{G}$ (resp., $\widehat{G}_{f}$ or $\widehat{G}_{f f}$ ) by $\widehat{G}^{\varphi}$ (resp., $\widehat{G}_{f}^{\varphi}$ or $\widehat{G}_{f f}^{\varphi}$ ).

In some important cases these subspaces coincide with the entire spaces:

Proposition 2.3. (1) If $G$ is abelian, $\widehat{G}=\widehat{G}_{f}=\widehat{G}^{\varphi}=\widehat{G}_{f}^{\varphi}$.

(2) If $\varphi$ is an epimorphism, $\widehat{G}=\widehat{G}^{\varphi}$ and $\widehat{G}_{f}=\widehat{G}_{f}^{\varphi}$

Proof. The first statement immediately follows from the fact that a representation of an abelian group is irreducible if and only if it is 1-dimensional.

The second one follows from Lemma 1.7 keeping in mind that $\varphi^{n}$ is an epimorphism if and only if $\varphi$ is.

Evidently continuous (w.r.t. the topology of weak containment) maps $\widehat{\varphi^{n}}:[\rho] \mapsto\left[\rho \circ \varphi^{n}\right]$ are defined for these subspaces $\widehat{G}^{\varphi}, \widehat{G}_{f}^{\varphi}$, and $\widehat{G}_{f f}^{\varphi}$ (generally not a homeomorphism!) and $\widehat{\varphi^{n}}=(\widehat{\varphi})^{n}$. Thus we obtain a dynamical system as the corresponding action of the semigroup $\mathbb{N}_{0}=\{0,1,2, \ldots\}$ (we will reserve $\mathbb{N}$ for $\{1,2, \ldots\}$ ).

The key observation is the following one:

Lemma 2.4. Let $[\rho] \in \widehat{G}$ be an $\widehat{\varphi}^{n}$-f-point for some $n \geq 1$, i.e. the representations $\rho$ and $\rho \circ \varphi^{n}$ are equivalent. Then $[\rho] \in \widehat{G}^{\varphi}$.

Proof. By Lemma 1.8 we obtain

$$
\rho \sim \rho \circ \varphi^{n} \sim \cdots \sim \rho \circ \varphi^{k n} \sim \ldots
$$

In particular, all they are irreducible. Now consider an arbitrary $m$ and choose $k$ such that $m \leq k n$. Then $\operatorname{Im} \rho \circ \varphi^{m} \supseteq \operatorname{Im} \rho \circ \varphi^{k n}$ and $\left(\operatorname{Im} \rho \circ \varphi^{m}\right)^{\star} \subseteq\left(\operatorname{Im} \rho \circ \varphi^{k n}\right)^{\star}$. It remains to apply Lemma 1.7 and conclude that $\rho \circ \varphi^{m}$ is irreducible.

Corollary 2.5. So, we have no dynamical system generated by $\varphi$ on $\widehat{G}$ (resp, $\widehat{G}_{f}$, or $\widehat{G}_{f f}$ ) generally, but we have the well-defined notion of a $\widehat{\varphi}^{n}$-f-point.

The corresponding well-defined dynamical system exists on $\widehat{G}^{\varphi}$ (resp, $\widehat{G}_{f}^{\varphi}$, or $\widehat{G}_{f f}^{\varphi}$ ) and its $n$-periodic points are exactly $\widehat{\varphi}^{n}$-f-points.

Definition 2.6. Denote the number of $\widehat{\varphi}^{n}$-f-points by $\mathbf{F}\left(\widehat{\varphi}^{n}\right)$.

\section{TBFT IMPLIES CONGRUENCES}

Definition 3.1. We say that TBFT (resp., TBFT, TBFT $T_{f}$ ) takes place for an endomorphism $\varphi: G \rightarrow G$ and its iterations, if $R\left(\varphi^{n}\right)<\infty$ and $R\left(\varphi^{n}\right)$ coincides with the number of $\widehat{\varphi}^{n}$-f-points in $\widehat{G}$ (resp., in $\widehat{G}_{f}, \widehat{G}_{f f}$ ) for all $n \in \mathbb{N}$.

Similarly, one can give a definition for a single endomorphism (without iterations).

Definition 3.2. Denote by $\mu(d), d \in \mathbb{N}$, be the Möbius function, i.e.

$$
\mu(d)= \begin{cases}1 & \text { if } d=1, \\ (-1)^{k} & \text { if } d \text { is a product of } k \text { distinct primes } \\ 0 & \text { if } d \text { is not square }- \text { free. }\end{cases}
$$


Theorem 3.3. Suppose, TBFT (resp., TBFT or TBFT $T_{f}$ ) takes place for an endomorphism $\varphi: G \rightarrow G$ and its iterations. In particular, $R\left(\varphi^{n}\right)<\infty$ for any $n$. Then one has the following Gauss congruences for Reidemeister numbers:

$$
\sum_{d \mid n} \mu(d) \cdot R\left(\phi^{n / d}\right) \equiv 0 \bmod n
$$

for any $n$.

Proof. This follows from 2.5 and the general theory of congruences for periodic points (cf. $[43,47])$.

More precisely, let $P_{n}$ be the number of periodic points of least period $n$ of the dynamical system of 2.5. Then $R\left(\varphi^{n}\right)=\mathbf{F}\left(\widehat{\varphi}^{n}\right)=\sum_{d \mid n} P_{d}$. By the Möbius inversion formula,

$$
\sum_{d \mid n} \mu(d) R\left(\varphi^{n / d}\right)=P_{n} \equiv 0 \bmod n
$$

since each orbit brings to $P_{n}$ just $n$ points.

Remark 3.4. In [22] it is shown that $\mathrm{TBFT}_{f}$ and $\mathrm{TBFT}_{f f}$ are equivalent for finitely generated groups (see also [46]).

\section{TBFT FOR ENDOMORPHISMS OF ABELIAN AND FINITE GROUPS}

For these classes all irreducible representations are finite-dimensional. That is why TBFT is the same as $\mathrm{TBFT}_{f}$. In [8] using Pontryagin duality the following statement was proved.

Proposition 4.1. TBFT $T_{f}$ holds for any endomorphism of an abelian group.

For polycyclic groups below we need also

Proposition 4.2. TBFT $T_{f}$ holds for any endomorphism of an abelian group.

Proof. Indeed, in an abelian group

$$
\begin{gathered}
x \varphi\left(x^{-1}\right) y \varphi\left(y^{-1}\right)=(x y) \varphi\left((x y)^{-1}\right), \quad\left(x \varphi\left(x^{-1}\right)\right)^{-1}=x^{-1} \varphi(x), \\
x g \varphi\left(x^{-1}\right)\left(y g \varphi\left(y^{-1}\right)\right)^{-1}=\left(x y^{-1}\right) \varphi\left(\left(x^{-1} y\right)\right) .
\end{gathered}
$$

This shows that the Reidemeister class of $e$ is a subgroup $H$, and the other classes are $H$-cosets. Being a Reidemeister class, $H$ is $\varphi$-invariant (see Lemma 1.10 above) and the factorization $p: G \rightarrow G / H$ gives a bijection of Reidemeister classes. The induced action on $G / H$ is trivial, as well as on $\widehat{G / H}$. The fixed representations $\rho \circ p$, where $\rho$ runs over $\widehat{G / H}$, are desired finite representations.

Theorem 4.3 (cf. [8]). Let $\varphi: G \rightarrow G$ be an endomorphism of a finite group $G$. Then the Reidemeister number $R(\varphi)$ coincides with the number of $\widehat{\varphi}-\mathbf{f}$-points on $\widehat{G}$, i.e. TBFT is true in this situation.

Proof. Let us note that $R(\varphi)$ is equal to the dimension of the space of $\varphi$-class functions (i.e. those functions that are constant on Reidemeister classes). They can be also described as fixed elements of the action $a \mapsto g a \varphi\left(g^{-1}\right)$ on the group algebra $\mathbb{C}[G]$. For the latter algebra we have the Peter-Weyl decomposition

$$
\mathbb{C}[G] \cong \bigoplus_{[\rho] \in \widehat{G}} \text { End } V_{\rho}, \quad \rho: G \rightarrow U\left(V_{\rho}\right),
$$


which respects the left and right $G$-actions. Hence,

$$
R(\varphi)=\sum_{[\rho] \in \widehat{G}} \operatorname{dim} T_{\rho}, \quad T_{\rho}:=\left\{a \in \text { End } V_{\rho} \mid a=\rho(g) a \rho\left(\varphi\left(g^{-1}\right) \text { for all } g \in G\right\} .\right.
$$

Thus, if $0 \neq a \in T_{\rho}, a$ is an intertwining operator between the irreducible representation $\rho$ and some representation $\rho \circ \varphi$. This implies that $\rho$ is equivalent to some (irreducible) subrepresentation $\pi$ of $\rho \circ \varphi$ (cf. [35, VI, p.57]). Hence, $\operatorname{dim} \rho=\operatorname{dim} \pi$, while $\operatorname{dim} \rho=\operatorname{dim} \rho \circ \varphi$. Thus, $\pi=\rho \circ \varphi$, and is irreducible. In this situation $\operatorname{dim} T_{\rho}=1$ by the Schur lemma. Evidently, vice versa, if $\rho \sim \rho \circ \varphi$ then $\operatorname{dim} T_{\rho}=1$. Hence,

$$
R(\varphi)=\sum_{[\rho] \in \widehat{G}}\left\{\begin{array}{l}
1, \text { if } \rho \sim \rho \circ \varphi \\
0, \text { if } \rho \nsim \rho \circ \varphi
\end{array}=\text { number of } \widehat{\varphi}\right. \text {-f-points. }
$$

\section{TECHNICAL LEMMAS}

We will need the following

Lemma 5.1. Let $\rho$ be a finite representation. It is a $\widehat{\varphi}$-f-point if and only if there exists a non-zero $\varphi$ class function being a matrix coefficient of $\rho$.

In this situation this function is unique up to scaling and is defined by the formula

$$
T_{S, \rho}: g \mapsto \operatorname{Tr}(S \circ \rho(g))
$$

where $S$ is an intertwining operator between $\rho$ and $\rho \circ \varphi$ :

$$
\rho(\varphi(x)) S=S \rho(x) \quad \text { for any } x \in G .
$$

In particular, $\mathrm{TBFT}_{f f}$ is true for $\varphi$ if and only if the above matrix coefficients form a base of the space of $\varphi$-class functions.

Proof. First, let us note that (1) defines a class function:

$$
T_{S, \rho}\left(x g \varphi\left(x^{-1}\right)\right)=\operatorname{Tr}\left(S \rho\left(x g \varphi\left(x^{-1}\right)\right)\right)=\operatorname{Tr}\left(\rho(\varphi(x)) S \rho(g) \rho\left(\varphi\left(x^{-1}\right)\right)=\operatorname{Tr}(S \rho(g)) .\right.
$$

If $S \neq 0$, then $\rho(a)=S^{*}$ for some $a \in \ell^{1}(G)$, and $\operatorname{Tr}\left(S S^{*}\right) \neq 0$. Thus, the $\varphi$-class function is non-zero. On the other hand, any matrix coefficient of $\rho$, i.e. a functional $T: \operatorname{End}\left(V_{\rho}\right) \rightarrow \mathbb{C}$ has the form $g \mapsto \operatorname{Tr}(D \rho(g))$ for some fixed matrix $D \neq 0$. If it is a $\varphi$-class function, then for any $g \in G$, or similarly, $a \in \ell^{1}(G)$,

$$
\operatorname{Tr}(D \rho(a))=\operatorname{Tr}\left(D \rho\left(x a \varphi\left(x^{-1}\right)\right)\right)=\operatorname{Tr}\left(\rho\left(\varphi\left(x^{-1}\right)\right) D \rho(x) \rho(a)\right) .
$$

Since $\rho(a)$ runs over all the matrix algebra, this implies $D=\rho\left(\varphi\left(x^{-1}\right)\right) D \rho(x)$, or $\rho(\varphi(x)) D=$ $D \rho(x)$, i.e. $D$ is the desired non-zero intertwining operator.

The uniqueness up to scaling follows now from the explicit formula and the Shur lemma.

The last statement follows from linear independence of matrix coefficients of non-equivalent representations.

Lemma 5.2. A group $G$ satisfies $T B F T_{f f}$ for an endomorphism $\varphi: G \rightarrow G$ if and only if there exists a $\varphi$-equivariant factorization of $G$ onto a finite group $F$, such that Reidemeister classes on $G$ map onto distinct classes on $F$. 
Proof. Let $\rho_{1}, \ldots \rho_{k}$ be all finite $\widehat{\varphi}$-f-representations, $F_{1}, \ldots F_{k}, F_{i}=\rho_{i}(G)$ the corresponding finite groups. Suppose, $g \in \operatorname{Ker} \rho_{i}=: K_{i}$. Then $\rho(\varphi(g))=S \rho(g) S^{-1}=S S^{-1}=e$. Hence, $K_{i}$ is a normal $\varphi$-invariant subgroup. Define $K:=\cap K_{i}$. It is still a normal $\varphi$-invariant subgroup of finite index. Let $F:=G / K$. By Lemma 1.9 the Reidemeister number of the induced map $\varphi_{F}$ satisfies $R\left(\varphi_{F}\right) \leq R(\varphi)$. On the other hand, $\rho_{i}$ define disjoint irreducible representations of $F$ corresponding to $\widehat{\varphi}_{F}$-f-points on $\widehat{F}$. By Lemma 5.1 this implies $R\left(\varphi_{F}\right) \geq R(\varphi)$. Thus, $G \rightarrow F$ gives a bijection of Reidemeister classes.

The opposite statement follows from Lemma 5.1 and Theorem 4.3. Indeed, if $p: G \rightarrow F$ is the mentioned epimorphism with $r=R(\varphi)=R\left(\varphi_{F}\right)$, then by Theorem 4.3 we have exactly $r$ pairwise non-equivalent irreducible unitary $\widehat{\varphi_{F}}$-f-representations $\rho_{1}, \ldots, \rho_{r}$ of $F$. Then $\rho_{i} \circ p$ play the same role for $G$. Finally $G$ can not have "additional" $\widehat{\varphi}$-f-representations by linear independence in Lemma 5.1.

We can enforce this statement.

Lemma 5.3. Let $\varphi: G \rightarrow G$ be an endomorphism with $R(\varphi)<\infty$. Suppose there exists $a$ (not necessarily equivariant) factorization $p$ of $G$ onto a finite group $F$, such that Reidemeister classes on $G$ map bijectively onto some disjoint subsets (not necessarily classes) on $F$. Then $G$ has TBFT $_{\text {ff }}$ for $\varphi$.

Proof. For $F$, characteristic functions of any sets are matrix coefficients of some (finite) representations. Thus, characteristic functions of Reidemeister classes on $G$ are matrix coefficients of some finite representations (coming from $F$ ). Lemma 5.1 completes the proof.

Definition 5.4. We will say under the supposition of Lemma 5.3 that $p$ separates Reidemeister classes. Similarly, we will say that $p$ separates some classes if it maps them to disjoint subsets.

Lemma 5.5. Let $\varphi$ be an endomorphism of a group $G$ with $R(\varphi)<\infty$. Then $G$ has TBFT $_{f f}$ for $\varphi$ if and only if the right shifts of all Reidemeister classes form a finite number of subsets of $G$.

Proof. First, let us note, that if we have a bijection for Reidemeister classes of $\varphi$, then we have a bijection for Reidemeister classes of any $\tau_{g} \circ \varphi$ by Lemma 1.12 .

Now the "only if" direction is evident, because these sets are pre-images of some sets in finite $F$.

Suppose, there are finitely many shifts. This means, that the stabilizers under right shifts of any Reidemeister class of $\varphi$ have finite index. Since $R(\varphi)<\infty$, their intersection is a subgroup $H$ in $G$ of finite index. By Lemma 1.12, its elements stabilize Reidemeister classes of any $\tau_{g} \circ \varphi$.

Suppose, $h \in H$, then

$$
\begin{aligned}
y g \varphi\left(y^{-1}\right) z h z^{-1} & =y(g z) z^{-1} \varphi\left(y^{-1}\right) z h z^{-1}=y(g z)\left(\tau_{z^{-1}} \circ \varphi\right)\left(y^{-1}\right) h z^{-1} \\
& =x(g z)\left(\tau_{z^{-1}} \circ \varphi\right)\left(x^{-1}\right) z^{-1}=x g \varphi\left(x^{-1}\right)
\end{aligned}
$$

for some $x$. Thus, $H$ is normal (and $\varphi$-invariant, if $\varphi$ is an automorphism). The projection $G \rightarrow G / H=: F$ maps Reidemeister classes of $\varphi$ bijectively onto disjoint sets in $F$. Indeed, if $h \in H$, then for any $g$,

$$
e \cdot h=x e\left(\tau_{g} \circ \varphi\right)\left(x^{-1}\right) .
$$


This means, that $H$ entirely is in the Reidemeister class of $e$ of any $\tau_{g} \circ \varphi$. By Lemma 1.12 this means that all Reidemeister classes of $\phi$ are formed by $H$-cosets and we are done.

It remains to apply Lemma 5.3.

Lemma 5.6. Suppose, $G$ has only finitely many inner automorphisms and an endomorphism $\varphi: G \rightarrow G$ with $R(\varphi)<\infty$. Then $G$ has $\operatorname{TBFT}_{f f}$ for $\varphi$.

Proof. By Lemma 1.12, the number of right shifts of Reidemeister classes is not more than $R(\phi) \cdot|\operatorname{Inn}(G)|$. It remains to apply Lemma 5.5.

Lemma 5.7. Let $\varphi: A \rightarrow A$ be an endomorphism of a finitely generated abelian group $A$ with $R(\varphi)<\infty$. Then the number of fixed elements $C(\varphi)$ on $A$ is finite.

Proof. The torsion subgroup is finite and totally invariant. Factorizing we reduce the problem to the case of $\mathbb{Z}^{n}$ by Lemma 1.9. In this case we will show that $\varphi$ has only the trivial fixed element 0. By [8] in this case $\operatorname{det}(\operatorname{Id}-\varphi) \neq 0$, considered as $n \times n$ integer matrix. Diagonalising this matrix by left and right multiplication by unimodular matrix (as it was done e.g. in [3]) we see that it can not have (non-zero) eigenvector with eigenvalue zero, i.e. there is no non-trivial $\varphi$-fixed point.

\section{6. $\mathrm{TBFT}_{f f}$ FOR ENDOMORPHISMS OF POLYCICLIC GROUPS}

Consider a polycyclic group $G$. Its (finite) derived series is formed by fully invariant subgroups $G_{i}$ with abelian quotients $A_{i}=G_{i} / G_{i+1}$. The key difference from a general finitely generated solvable group is the following: all $G_{i}$ and $A_{i}$ are finitely generated (see [38] for details). Let $G_{n} \neq\{e\}, G_{n+1}=\{e\}$.

We will argue by induction. For the basis of this induction let us observe that for the abelian group $G_{n}$ and any its endomorphism with finite Reidemeister number we have $\mathrm{TBFT}_{f f}$ by Prop. 4.1. Now suppose by induction that same is true for $G_{i+1}$ and prove it for $G_{i}$. Denote $G_{i+1}=: H, G_{i}=: \Gamma, A_{i}=: A$.

Consider an endomorphism $\varphi: \Gamma \rightarrow \Gamma$ with $R(\varphi)<\infty$ and induced endomorphisms $\varphi_{H}: H \rightarrow H$ and $\varphi_{A}: A \rightarrow A$. Then by Lemma $1.9 R\left(\varphi_{A}\right)<\infty$ and by Lemma 5.7 the number of fixed elements of $\varphi_{A}$ on $A$ is finite. Thus, by Lemma $1.11 R\left(\varphi_{H}\right)<\infty$. Let $H_{0} \subset H$ be a normal $\varphi$-invariant subgroup of finite index such that $H \rightarrow H / H_{0}$ gives a bijection on Reidemeister classes (see Lemma 5.2). This means that classes of $\varphi_{H}$ are some unions of $H_{0}$ cosets. Consider automorphisms $\tau_{g}: H \rightarrow H, \tau_{g}(h)=g h g^{-1}, g \in \Gamma$, and define $H_{1}:=\cap_{g \in \Gamma} \tau_{g}\left(H_{0}\right)$. All subgroups in the intersection have the same finite index in $H$. Since $H$ is finitely generated, $H_{1}$ also has a finite index. By construction, $H_{1}$ is normal in $\Gamma$. Also,

$$
\varphi\left(\tau_{g}\left(h_{0}\right)\right)=\varphi(g) \varphi\left(h_{0}\right)(\varphi(g))^{-1}=\tau_{\varphi(g)}\left(\varphi\left(h_{0}\right)\right), \quad h_{0}, \varphi\left(h_{0}\right) \in H_{0},
$$

i.e. $\varphi\left(\tau_{g}\left(H_{0}\right)\right) \subset \tau_{\varphi(g)}\left(H_{0}\right)$. Hence, $H_{1}$ is $\varphi$-invariant and $H_{1} \subset H_{0}$, thus $H_{1}$ can play the same role as $H_{0}$ with an additional property of being normal in $\Gamma$. In particular, classes of $\varphi_{H}$ are some unions of $H_{1}$ cosets. The same is true for intersections of Reidemeister classes of $\varphi$ with $H$ (because they are unions of some classes of $\varphi_{H}$ ). This means that $\Gamma \rightarrow \Gamma / H_{1}$ separates these classes, i.e. the classes which map on the Reidemeister class of $e \in A$ under $\Gamma \rightarrow A$. Similarly we can find subgroups $H_{2}, \ldots H_{R\left(\varphi_{A}\right)}$ which separate classes over other classes (i.e. which are mapped onto other classes) of $A$. For this purpose, suppose $g \in \Gamma$ is over some other class of $A$. Then the classes of $\tau_{g} \circ \varphi$ (with the same finite Reidemeister number) that intersect with $H$ are obtained from the classes under consideration by a shift by $g$ (see Lemma 1.12). Then a group $H_{2}=H_{1}^{g}$ constructed for $\left.\tau_{g} \circ \varphi\right|_{H}$ in the same way as 
$H_{1}$ for $\varphi_{H}$, will separate these classes. Moreover, it will be $\tau_{g} \circ \varphi$-invariant for this specific $g$ and normal in $\Gamma$, i.e. $\tau_{g^{\prime}}$-invariant for any $g^{\prime} \in \Gamma$. Taking $g^{\prime}=g^{-1}$ we see that $H_{1}^{g}$ is $\varphi$-invariant. Similarly we define the other $H_{i}$ choosing $g$ over other Reidemeister classes of $\varphi_{A}$. Taking the (finite!) intersection of $H_{1}, H_{2}, \ldots H_{R\left(\varphi_{A}\right)}$ we obtain a $\varphi$-invariant subgroup of finite index $H^{\prime} \subset H$, which is normal in $\Gamma$, and $p: \Gamma \rightarrow \Gamma / H^{\prime}=\Gamma^{\prime}$ gives a bijection on Reidemeister classes; $F:=p(H)=H / H^{\prime} \subset \Gamma^{\prime}$ is a finite normal subgroup and $\Gamma^{\prime} / F \cong A$ :

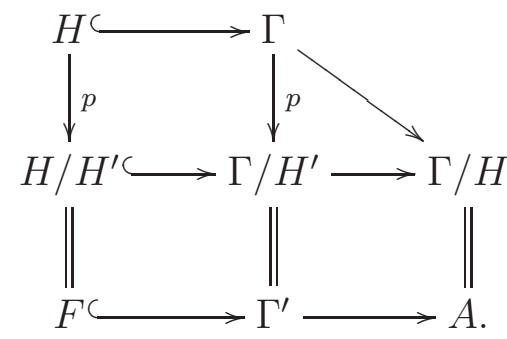

Thus, $\Gamma^{\prime}$ is finitely generated finite-by-abelian group, and it has finitely many inner automorphisms. Lemma 5.6 completes the proof of the following statement.

Theorem 6.1. Let $\varphi: G \rightarrow G$ be an endomorphism of a polycyclic group with $R(\varphi)<\infty$. Then $T B F T_{f f}$ is true for $\varphi$.

Remark 6.2. The results of this section can be extended to some virtually polycyclic groups (under supposition of some polycyclic subgroup to be $\varphi$-invariant).

More precisely the following statement can be proved by word-by-word rewriting of the above argument.

Theorem 6.3. Let $G$ be an almost polycyclic group admitting a fully invariant polycyclic subgroup of finite index. Then TBFT ff is true for any endomorphism $\varphi: G \rightarrow G$ with $R(\phi)<\infty$.

Let us illustrate this theorem by the following

Example 6.4. In [28] seven series of almost polycyclic (polycyclic-by-finite) groups with the property $R_{\infty}$ (any automorphism has infinite Reidemeister number) were found. They have a fully invariant polycyclic subgroup of finite index ([28, Remark 2.1], [32]). Thus, they are covered by Theorem 6.3.

On the other hand, they evidently have endomorphisms with finite Reidemeister number (see Prop. 1.1 and Cor. 1.4).

\section{REFERENCES}

[1] J. Arthur And L. Clozel. Simple algebras, base change, and the advanced theory of the trace formula. Princeton University Press, Princeton, NJ, 1989.

[2] Collin Bleak, Alexander Fel'shtyn, and Daciberg L. Gonçalves. Twisted conjugacy classes in R. Thompson's group F. Pacific J. Math. 238, No. 1, 1-6, 2008.

[3] Robin B. S. Brooks, Robert F. Brown, Jingyal Pak, and Douglas H. Taylor. Nielsen numbers of maps of tori. Proc. Amer. Math. Soc. 52, 398-400, 1975.

[4] Karel Dekimpe and Daciberg Gonçalves. The $R_{\infty}$ property for free groups, free nilpotent groups and free solvable groups. Bull. Lond. Math. Soc. 46, No. 4, 737-746, 2014.

[5] Alexander Fel/shtyn and Richard Hill. Trace formulae, zeta functions, congruences and Reidemeister torsion in Nielsen theory. Forum Math. 10, No. 6, 641-663, 1998.

[6] A. FeL'shtyn. Dynamical zeta functions, Nielsen theory and Reidemeister torsion. Mem. Amer. Math. Soc. 147, No. 699, xii+146, 2000. 
[7] A. Fel'shtyn. The Reidemeister number of any automorphism of a Gromov hyperbolic group is infinite. Zap. Nauchn. Sem. S.-Peterburg. Otdel. Mat. Inst. Steklov. (POMI) 279, No. 6 (Geom. i Topol.), 229$240,250,2001$.

[8] A. Fel'shtyn and R. Hill. The Reidemeister zeta function with applications to Nielsen theory and a connection with Reidemeister torsion. K-Theory 8, No. 4, 367-393, 1994.

[9] A. Fel'shtyn, F. Indukaev, and E. Troitsky. Twisted Burnside theorem for two-step torsion-free nilpotent groups. In $C^{*}$-algebras and elliptic theory. II, Trends in Math., pages 87-101. Birkhäuser, 2008.

[10] A. Fel'shtyn, Yu. Leonov, and E. Troitsky. Twisted conjugacy classes in saturated weakly branch groups. Geometriae Dedicata 134, 61-73, 2008.

[11] A. Fel'shtyn, N. Luchnikov, and E. Troitsky. Reidemeister classes and twisted inner representations. Russ. J. Math. Phys. 22, No. 3, 301-306, 2015.

[12] A. Fel'shtyn and E. Troitsky. A twisted Burnside theorem for countable groups and Reidemeister numbers. In C. Consani and M. Marcolli, editors, Noncommutative Geometry and Number Theory, pages 141-154. Vieweg, Braunschweig, 2006.

[13] A. Fel'Shtyn and E. Troitsky. Twisted Burnside-Frobenius theory for discrete groups. J. Reine Angew. Math. 613, 193-210, 2007.

[14] A. Fel'shtyn, E. Troitsky, And A. Vershik. Twisted Burnside theorem for type $\mathrm{II}_{1}$ groups: an example. Math. Res. Lett. 13, No. 5, 719-728, 2006.

[15] Alexander Fel'shtyn. New directions in Nielsen-Reidemeister theory. Topology Appl. 157, No. 10-11, $1724-1735,2010$.

[16] Alexander Fel'shtyn and Daciberg L. Gonçalves. The Reidemeister number of any automorphism of a Baumslag-Solitar group is infinite. In Geometry and dynamics of groups and spaces, volume 265 of Progr. Math., pages 399-414. Birkhäuser, Basel, 2008.

[17] Alexander Fel'shtyn and Daciberg L. Gonçalves. Twisted conjugacy classes in symplectic groups, mapping class groups and braid groups. Geom. Dedicata 146, 211-223, 2010. With an appendix written jointly with Francois Dahmani.

[18] Alexander Fel'shtyn and Daciberg L. Gonçalves. Reidemeister spectrum for metabelian groups of the form $Q^{n} \rtimes \mathbb{Z}$ and $\mathbb{Z}[1 / p]^{n} \rtimes \mathbb{Z}, p$ prime. Internat. J. Algebra Comput. 21, No. 3, 505-520, 2011.

[19] Alexander Fel'shtyn and Jong Bum Lee. The Nielsen and Reidemeister numbers of maps on infra-solvmanifolds of type (R). Topology Appl. 181, 62-103, 2015.

[20] Alexander Fel'shtyn and Timur Nasybullov. The $R_{\infty}$ and $S_{\infty}$ properties for linear algebraic groups. J. Group Theory 19, No. 5, 901-921, 1916.

[21] Alexander Fel'shtyn and Evgeniu Troitsky. Geometry of Reidemeister classes and twisted Burnside theorem. J. K-Theory 2, No. 3, 463-506, 2008.

[22] Alexander Fel'shtyn and Evgeniu Troitsky. Aspects of the property $R_{\infty}$. J. Group Theory 18, No. 6, 1021-1034, 2015.

[23] Felix Gantmacher. Canonical representation of automorphisms of a complex semi-simple Lie group. Rec. Math. (Moscou) 5(47), 101-146, 1939.

[24] D. GonçALves. The coincidence Reidemeister classes on nilmanifolds and nilpotent fibrations. Topology and Its Appl. 83, 169-186, 1998.

[25] Daciberg Gonçalves and Dessislava Hristova Kochloukova. Sigma theory and twisted conjugacy classes. Pacific J. Math. 247, No. 2, 335-352, 2010.

[26] Daciberg Gonçalves and Peter Wong. Twisted conjugacy classes in nilpotent groups. J. Reine Angew. Math. 633, 11-27, 2009.

[27] A. Grothendieck. Formules de Nielsen-Wecken et de Lefschetz en géométrie algébrique. In Séminaire de Géométrie Algébrique du Bois-Marie 1965-66. SGA 5, volume 569 of Lecture Notes in Math., pages 407-441. Springer-Verlag, Berlin, 1977.

[28] Ku Yong Ha And Jong Bum Lee. The $R_{\infty}$ property for crystallographic groups of Sol. Topology Appl. 181, 112-133, 2015.

[29] B. Jiang. Lectures on Nielsen Fixed Point Theory, volume 14 of Contemp. Math. Amer. Math. Soc., Providence, RI, 1983.

[30] B. JiAnG. Fixed points and braids. Invent. Math. 75, No. 1, 69-74, 1984. 
[31] A. A. Kirillov. Elements of the Theory of Representations. Springer-Verlag, Berlin Heidelberg New York, 1976.

[32] Jong Bum Lee And Kyung Bai Lee. Averaging formula for Nielsen numbers of maps on infrasolvmanifolds of type (R). Nagoya Math. J. 196, 117-134, 2009.

[33] G. Levitt and M. Lustig. Most automorphisms of a hyperbolic group have very simple dynamics. Ann. Scient. Éc. Norm. Sup. 33, 507-517, 2000.

[34] T. Mubeena and P. Sankaran. Twisted conjugacy classes in lattices in semisimple lie groups. Transformation Groups 19, No. 1, 159-169, 2014.

[35] M. A. NAĬMARK And A. I. ŠTern. Theory of group representations, volume 246 of Grundlehren der Mathematischen Wissenschaften [Fundamental Principles of Mathematical Sciences]. Springer-Verlag, New York, 1982. Translated from the Russian by Elizabeth Hewitt, Translation edited by Edwin Hewitt.

[36] T. R. Nasybullov. Twisted conjugacy classes in general and special linear groups. Algebra Logika 51, No. 3, 331-346, 415, 418, 2012.

[37] A. L. Onishchik And È. B. Vinberg. Lie groups and algebraic groups. Springer Series in Soviet Mathematics. Springer-Verlag, Berlin, 1990. Translated from the Russian and with a preface by D. A. Leites.

[38] Derek J. S. Robinson. A course in the theory of groups, volume 80 of Graduate Texts in Mathematics. Springer-Verlag, New York, second edition, 1996.

[39] V. Roman'Kov. The twisted conjugacy problem for endomorphisms of polycyclic groups. J. Group Theory 13, No. 3, 355-364, 2010.

[40] V. Roman'kov. Twisted conjugacy classes in nilpotent groups. J. Pure Appl. Algebra 215, No. 4, 664-671, 2011.

[41] Jean-Pierre Serre. Galois cohomology. Springer Monographs in Mathematics. Springer-Verlag, Berlin, english edition, 2002. Translated from the French by Patrick Ion and revised by the author.

[42] Salahoddin Shokranian. The Selberg-Arthur trace formula, volume 1503 of Lecture Notes in Mathematics. Springer-Verlag, Berlin, 1992. Based on lectures by James Arthur.

[43] S. Smale. Differentiable dynamical systems. Bull. Amer. Math. Soc. 73, 747-817, 1967.

[44] T. A. Springer. Twisted conjugacy in simply connected groups. Transform. Groups 11, No. 3, 539$545,2006$.

[45] E. Troitsky. Noncommutative Riesz theorem and weak Burnside type theorem on twisted conjugacy. Funct. Anal. Pril. 40, No. 2, 44-54, 2006. In Russian, English translation: Funct. Anal. Appl. 40 (2006), No. 2, 117-125.

[46] Evgenij Troitsky. Two examples related to the twisted Burnside-Frobenius theory for infinitely generated groups. Fundam. Appl. Math. , 2017. (to appear).

[47] A. V. Zarelua. On congruences for the traces of powers of some matrices. Tr. Mat. Inst. Steklova 263, No. Geometriya, Topologiya i Matematicheskaya Fizika. I, 85-105, 2008. English translation: Proceedings of the Steklov Institute of Mathematics, 2008, Vol. 263, pp. 78-98.

Instytut Matematyki, Uniwersytet Szczecinski, Ul. Wielkopolska 15, 70-451 Szczecin, POLAND

E-mail address: felshtyn@mpim-bonn.mpg.de, fels@wmf.univ.szczecin.pl

Dept. of Mech. and Math., Moscow State University, 119991 GSP-1 Moscow, Russia

E-mail address: troitsky@mech.math.msu.su

$U R L:$ http://mech.math.msu.su/ troitsky 